\title{
The role of point-of-care ultrasound in intra-abdominal hypertension management
}

\author{
Bruno M. Pereira ${ }^{1,2}$, Renato G. Pereira ${ }^{2}$, Robert Wise ${ }^{3}$, Gavin Sugrue ${ }^{4}$, Tanya L. Zakrison ${ }^{5}$, \\ Alcir E. Dorigatti ${ }^{6}$, Rossano K. Fiorelli ${ }^{2}$, Manu L.N.G. Malbrain ${ }^{7-9}$ \\ ${ }^{1}$ President of the World Society of the Abdominal Compartment, University of Campinas, \\ Department of Surgery, Division of Trauma, Campinas, Brazil \\ 2Universidade Severino Sombra, Mestrado Profissional em Ciências Aplicadas em Saúde \\ ${ }^{3}$ Head Clinical Unit, Critical Care, Edendale Hospital, Pietermaritzburg, South Africa, \\ Discipline of Anaesthesiology and Critical Care, School of Clinical Medicine, University of KwaZulu-Natal, \\ Durban, South Africa \\ ${ }^{4}$ Department of Radiology, Mater Misericordiae University Hospital, Dublin, Ireland \\ ${ }^{5}$ Trauma Surgery and Surgical Critical Care, University of Miami, FL, USA \\ ${ }^{6}$ University of Campinas, Resident of the Department of Surgery, Campinas, Brazil \\ ${ }^{7}$ Department of Intensive Care Medicine and High Care Burn Unit Director, Ziekenhuis Netwerk Antwerpen, \\ ZNA Stuivenberg, Antwerp, Belgium \\ ${ }^{8}$ Department of Intensive Care Medicine, University Hospital Brussels (UZB), Jette, Belgium \\ ${ }^{9}$ Faculty of Medicine and Pharmacology, Free University Brussels (VUB), Brussels, Belgium
}

\begin{abstract}
Background: Intra-abdominal hypertension is a common complication in critically ill patients. Recently the Abdominal Compartment Society (WSACS) developed a medical management algorithm with a stepwise approach according to the evolution of the intra-abdominal pressure and aiming to keep IAP $\leq 15 \mathrm{~mm} \mathrm{Hg}$. With the increased use of ultrasound as a bedside modality in both emergency and critical care patients, we hypothesized that ultrasound could be used as an adjuvant point-of-care tool during IAH management. This may be particularly relevant to the first and second basic stages of the algorithm. The objective of this paper is to test the use of POCUS as an adjuvant tool in the management of patients with IAH/ACS. Methods: Seventy-three consecutive adult critically ill patients admitted to the surgical intensive care unit (ICU) of a single urban institution with risk factor for IAH/ACS were enrolled. Those who met the inclusion criteria were allocated to undergo POCUS as an adjuvant tool in their IAH/ACS management.
\end{abstract}

Results: A total of 50 patients met the inclusion criteria and were included in the study. The mean age of study participants was $55 \pm 22.6$ years, $58 \%$ were men, and the most frequent admission diagnosis was post-operative care following abdominal intervention. All admitted patients presented with a degree of IAH during their ICU stay. Following step 1 of the WSACS IAH medical management algorithm, ultrasound was used for NGT placement, confirmation of correct positioning, and evaluation of stomach contents. Ultrasound was comparable to abdominal X-ray, but shown to be superior in determining the gastric content (fluid vs. solid). Furthermore, POCUS allowed faster determination of correct NGT positioning in the stomach (antrum), avoiding bedside radiation exposure. Ultrasound also proved useful in: 1) evaluation of bowel activity; 2) identification of large bowel contents; 3 ) identification of patients that would benefit from bowel evacuation (enema) as an adjuvant to lower IAP; 4 ) and in the diagnosis of moderate to large amounts of free intra-abdominal fluid.

Conclusion: POCUS is a powerful systematic ultrasound technique that can be used as an adjuvant in intra-abdominal hypertension management. It has the potential to be used in both diagnosis and treatment during the course of IAH.

Anaesthesiology Intensive Therapy 2017, vol. 49, no 5, 373-381

Key words: intra-abdominal hypertension; intra-abdominal compartment syndrome; ultrasound; point-of-care ultrasound; POCUS 
The abdominal compartment is susceptible to wide ranging pressure variations. According to the Abdominal Compartment Society (WSACS, www.wsacs.org) 2013 consensus guidelines [1], normal intra-abdominal pressure in critically ill adults is regarded as 5-7 mm $\mathrm{Hg}$. Intra-abdominal hypertension (IAH) is defined by a sustained or repeatedly elevated pressure (> $12 \mathrm{~mm}$ $\mathrm{Hg}$ ) and has four grades: Grade I 12-15 mm Hg; Grade II 16-20 mm Hg; Grade III 21-25 mm Hg; Grade IV > 25 $\mathrm{mm}$ Hg. Recently, the Abdominal Compartment Society (WSACS) has developed a medical management algorithm with a stepwise approach based on the evolution of intra-abdominal pressure with the goal of keeping IAP $\leq 15 \mathrm{~mm} \mathrm{Hg}$ (level of evidence grade 1C) (Fig. 1). This algorithm is based on five basic principles, namely: 1 ) evacuation of intraluminal contents (e.g. stool, gastric residual volume); 2 ), evacuation of intra-abdominal contents (e.g. abscess, blood collection, ascites); 3) improvement of abdominal wall compliance; 4) optimization of fluid administration (neutral fluid balance); and 5) optimization of systemic and regional perfusion.

With the increased use of ultrasound [2] as a bedside modality in both emergency and critical care patients [3], we hypothesized that ultrasound could be used as an adjuvant point-of-care tool during IAH management. This may be particularly relevant to the first and second basic stages of the algorithm. The WSACS divides these two stages of IAH/ACS into 4 steps, as shown in Figure 1. The objective of this study was to test the use of POCUS as an adjuvant tool in the management of patients with IAH/ACS.

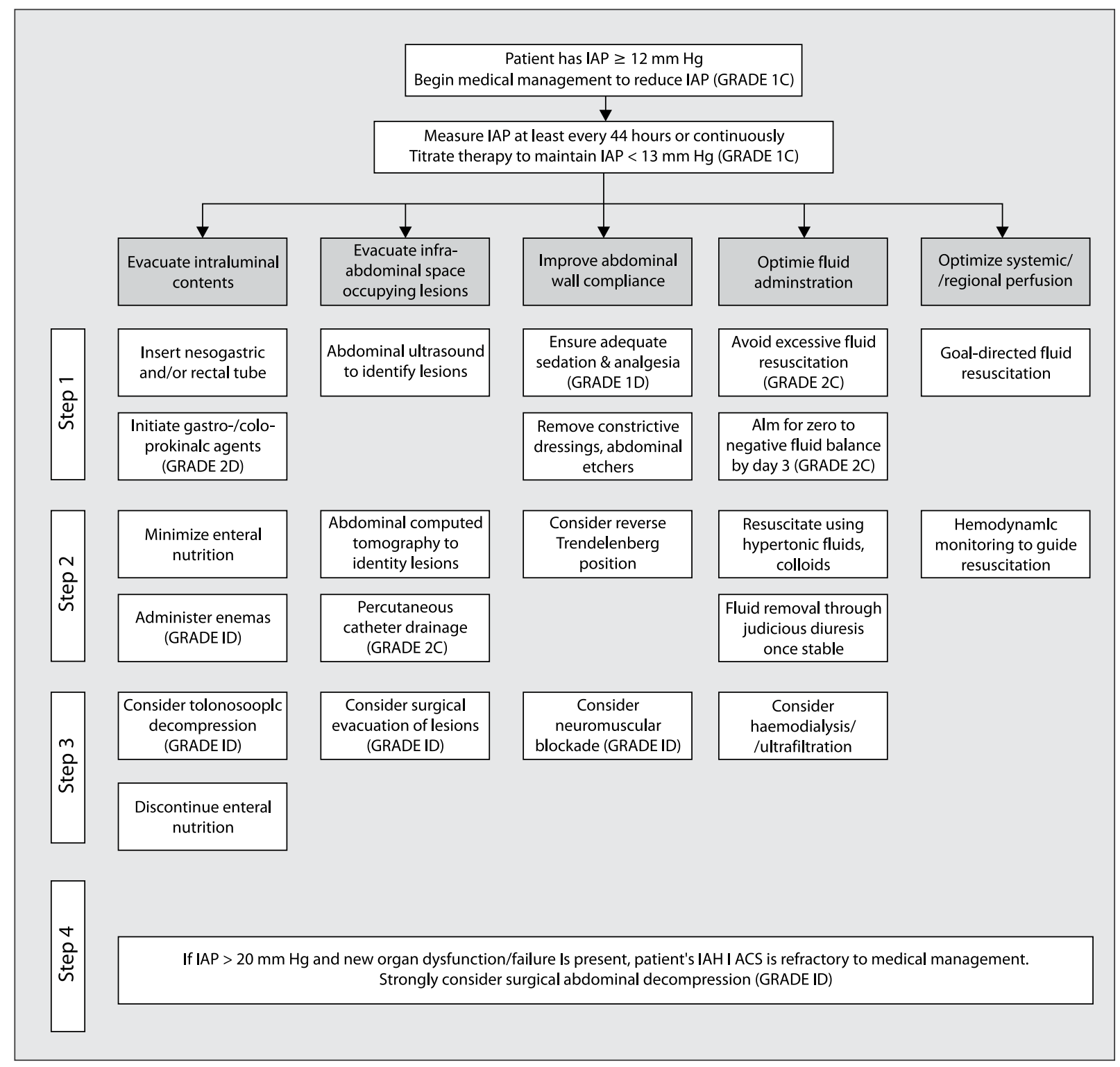

Figure 1. WSACS IAH/ ACS Medical Management Algorithm 


\section{METHODS \\ ETHICAL CONSIDERATIONS}

This IRB approved study (17031113.0.0000.5404) enrolled all adult critically ill patients admitted to the surgical intensive care unit (ICU) of a single urban institution from December $19^{\text {th }} 2016$ to February $28^{\text {th }} 2017$ with risk factors for IAH/ACS. Informed consent was waived, as there was no deviation from standard care and the WSACS medical management algorithm that was already adopted in the ICU.

\section{STUDY POPULATION}

All patients admitted with risk factors for IAH/ACS were included and treated according to the 2013 WSACS guidelines [1]. The inclusion criteria are shown in Table 1. Seventy-three consecutive patients were included in the study. A trained intensivist or surgeon performed POCUS for three consecutive days after admission:

1. When evacuation of intraluminal contents was indicated;

1.1. Ultrasound was used to confirm NGT position and compared to $\mathrm{X}$-ray imaging for patients requiring a nasogastric tube (NGT) for intra-abdominal decompression (WSACS algorithm step 1);

1.2. Stomach and bowel US was performed daily to evaluate hollow viscous content and/or enema effectiveness (WSACS algorithm step 2) and/or colonoscopy decompression (WSACS algorithm step 3);

2. When evacuation of intra-abdominal content was indicated;

2.1. Abdominal POCUS was performed daily, either to evaluate the presence of abdominal free fluid, or to help percutaneous drainage (WSACS algorithm step 2).

\section{IAP MEASUREMENT}

The IAP was measured according to the WSACS guidelines at end-expiration, with the patient in the supine position and the zero reference set at the level where the midaxillary line crosses the iliac crest. The IAP was either measured via the height of the urine column (Foley Manometer) or via a bedside monitor with a pressure transducer (AbViser', ConvaTec - São Paulo, Brazil).

\section{POCUS METHOD}

POCUS images were obtained in a systematic fashion with the patient in supine position, immediately after each 6-hour intra-abdominal pressure measurement, at endexpiration with adequate sedation, with or without the use of neuromuscular blocking drugs. A 64-element Mobissom (mobissom.com.br) M1 convex wireless ultrasound was used for all examinations (3.5 MHz, 90-200 mm, phased array).

For patients requiring NGT, images were obtained in B-mode with the transducer positioned at the level of the epi-
Table 1. Inclusion criteria adopted for the study

\begin{tabular}{ll}
\hline \multicolumn{2}{l}{ Inclusion criteria } \\
\hline A & ICU patients/ minimum ICU stay of 3 days \\
B & 18 years of age or older \\
C & Intubated and mechanically ventilated \\
D & Adequately sedated (RASS-4 or -5 ) \\
E & Able to lie in a supine position for all measurements \\
F & Undergoing treatment for IAH/ACS \\
G & Not exhibiting abdominal respiratory muscle activity \\
H & Not having a temporary open abdomen \\
I & Not exhibiting abdominal respiratory muscle activity
\end{tabular}

gastrium. First, ultrasound gel was liberally applied over the epigastrium. The convex transducer was placed in a transverse plane resulting in visualization of the antrum and body of the stomach. At this moment, insertion of the NGT was commenced and the stomach content was observed. Once the NGT was visible in the hollow viscous, a $20 \mathrm{~mL}$ flush of air was delivered to confirm correct positioning (Fig. 2).

Daily POCUS was performed in all patients to evaluate stomach and bowel content. For stomach views, the US window was used as described above. For small and large bowel visualization, the transducer was placed at the periumbilical level and on both medium-low abdominal quadrants to observe both the right and left colon.

To screen for intra-abdominal free fluid, the POCUS landmarks were the right upper quadrant, left upper quadrant and hypogastrium (Fig. 3), either with a longitudinal or transverse probe position. The various probe positions to enable the different POCUS windows is shown in Figure 4. Paracentesis was performed via the insertion of a sterile percutaneous needle with real-time direct ultrasound guidance.

\section{STATISTICAL ANALYSIS}

All demographic and clinical data were recorded prospectively in an Excel spreadsheet. A descriptive statistical analysis was performed to summarize patient characteristics and study measurements. Continuous variables are presented as the mean ( \pm standard deviation, SD) or median in the case of a skewed distribution. Categorical variables are expressed as numbers and percentages for the group from which they were derived. Continuous variables were compared with Student's t-test for normally distributed variables and the Mann-Whitney test for non-normally distributed variables. The $x^{2}$ test or Fisher's exact test were used to compare ordinal variables. All $p$-values are two-tailed and $P<0.05$ was considered statistically significant. Statistical analysis was performed with IBM ${ }^{\mathrm{TM}}$ SPSS (Windows version 21.0, 2016, Chicago, IL, USA). 


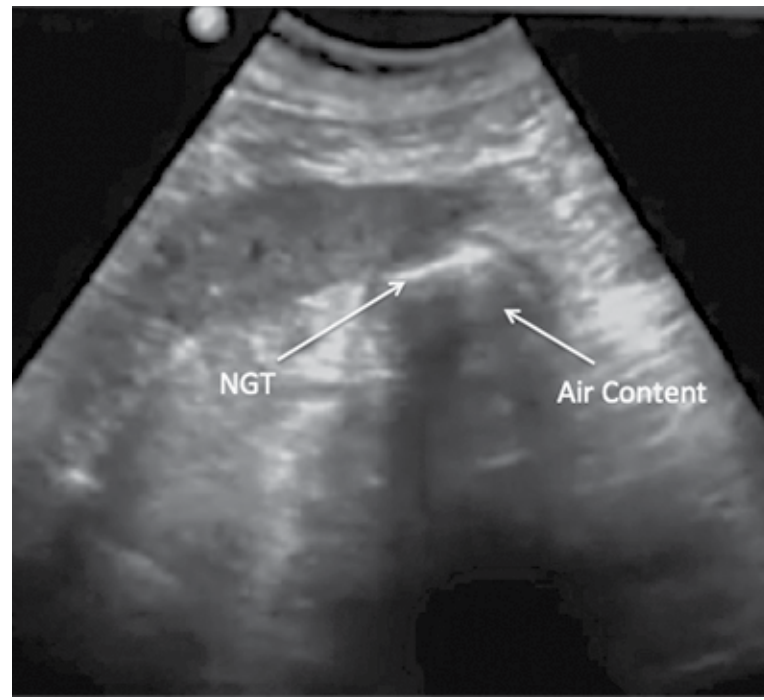

Figure 2. Nasogastric tube (NGT) ultrasound view at the moment of $20 \mathrm{~mL}$ flush of air

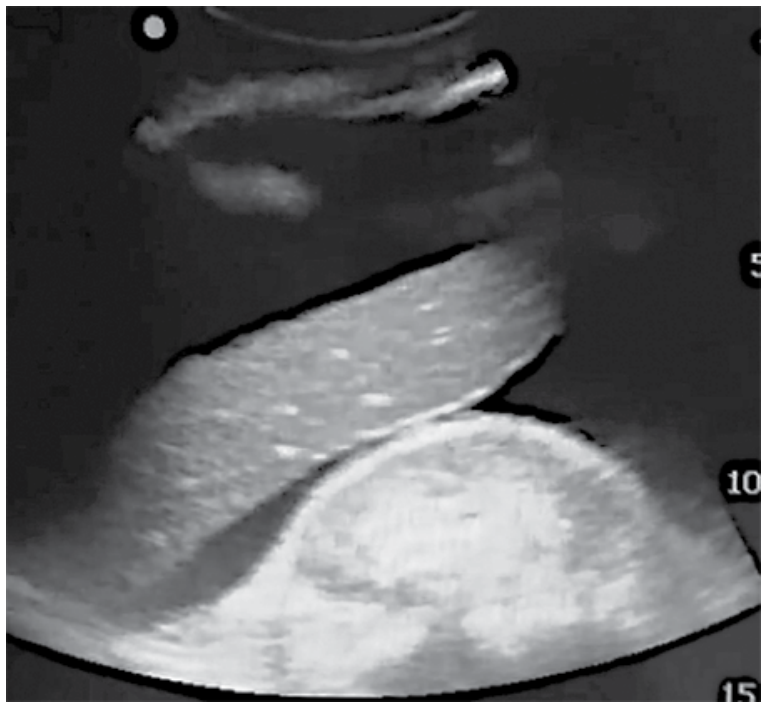

Figure 3. Right upper quadrant showing abdominal free fluid (ascites)

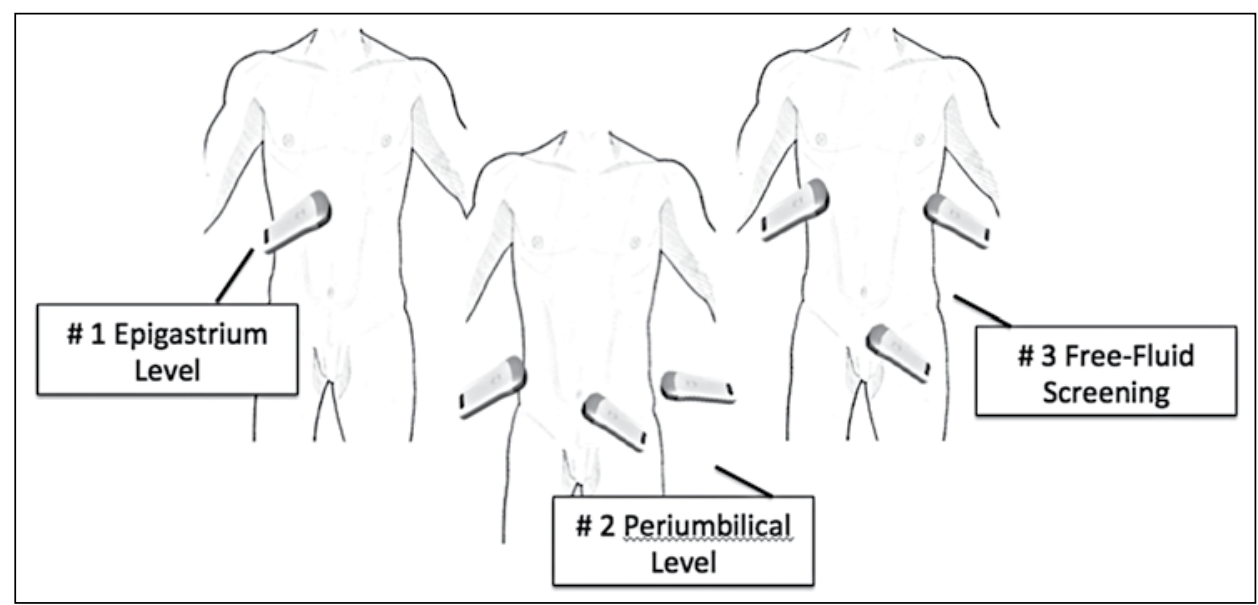

Figure 4. Probe position to access the different POCUS windows

\section{RESULTS}

A total of 73 patients were included in the study. Twenty-three patients were excluded due to one or more of the following reasons: death; extubation or discharge from ICU before the third day of admission; normal IAP; and the presence of an open abdomen. The mean age of study participants was $55 \pm 22.6$ years old, while $58 \%$ were men with one or more associated comorbidity such as hypertension, diabetes or dyslipidemia. The most frequent admission diagnosis was for post-operative care following abdominal intervention (Table 2). The majority of patients came from the emergency department (96\%). Table 2 shows the data from the first three consecutive ICU days. Decompressive laparotomy for raised IAP was not necessary in any of the patients due to full recovery after clinical management.
During the first three consecutive ICU days we observed a decrease in IAP with medical treatment. In general, patients were critically ill and $84 \%$ received vasoactive drugs. Mean IAP on admission was $23 \pm 15.5 \mathrm{~mm} \mathrm{Hg}$. Seventy-four percent of patients were admitted after surgery. All admitted patients presented with some degree of IAH/ACS during their ICU stay. Forty-six patients required a NGT for the first 48 hours following admission. Following step 1 of the WSACS medical management algorithm, ultrasound was used for NGT placement, confirmation of correct positioning, and to check stomach contents. Ultrasound was comparable to abdominal X-ray, but superior in determining gastric contents (fluid vs. solid). Furthermore, POCUS allowed faster bedside determination of correct NGT positioning into the stomach (antrum), without exposure to radiation. There was $100 \%$ accuracy when using US to determine NGT placement 
and positioning, with no false negatives or false positives observed. US also proved useful in patients on the third day of admission by confirming the safe removal of the NGT after screening demonstrated no gastric contents (Table 3 ).

The second step in the WSACS guidelines addresses intraluminal evacuation through the administration of enemas. This strategy was followed in all patients in whom the IAP remained high (above $20 \mathrm{~mm} \mathrm{Hg}$ ) on the second measurement ( 6 hours after admission). US proved useful in many ways: firstly, POCUS allowed assessment of bowel

Table 2. Patients characteristics, clinical data and admission diagnosis

\begin{tabular}{lc}
\hline Parameters & Participants (N = 50) \\
\hline Participant characteristics & $55(39-71)$ \\
Mean age (years) & $29(58 \%)$ \\
Gender (male) & 27 \\
BMI (kg m ${ }^{-2}$ ) & \\
Clinical data & $108.5(83-134)$ \\
Mean SBP (mm Hg) & $94(60-128)$ \\
Mean HR (beats min $\left.{ }^{-1}\right)$ & $50(100 \%)$ \\
IMV (\%) & $23(12-34)$ \\
Mean admission IAP (mm Hg) & 85 \\
Mean admission APP (mm Hg) & $42(84 \%)$ \\
Vasopressor use ( $\mathrm{n} \%)$ & \\
Admission diagnosis & $28(56 \%)$ \\
Bowell obstruction (\%) & $12(24 \%)$ \\
Abdominal sepsis (\%) & $8(16 \%)$ \\
Gastrointestinal bleeding (\%) & $2(4 \%)$ \\
Other (\%) & \\
\hline
\end{tabular}

activity (movements); secondly, it allowed identification of large bowel contents (right and left colon); and thirdly, POCUS allowed the identification of patients that may benefit from continued enema-treatment to lower IAP. These aspects were considered important, as the majority of patients were post-operative. For example, bowel movements were present on average 8 hours post-operatively, even with negative bowel sounds on auscultation. Enema treatment was found to empty the bowel incompletely in $72 \%, 56 \%$ and $42 \%$ of the times on days 1,2 and 3 respectively. Only one patient needed colonoscopic decompression, confirmed by US, clinically and with IAP improvement.

During the second stage of the WSACS medical management algorithm, US was a useful adjuvant tool for diagnosing moderate to large amounts of free intra-abdominal fluid. A small amount of fluid was expected as the majority of patients were coming from the OR. Special attention was given to cirrhotic patients that were admitted with upper gastrointestinal bleeding. Four patients in this group (out of a total of 8) were found to have large amounts of ascites and a US-guided paracentesis was carried out (Fig. 2). The average amount of ascites removed was $3600 \pm 1.6 \mathrm{~mL}$ and resulted in a significant drop in the IAP average from $21 \pm$ $4.1 \mathrm{~mm} \mathrm{Hg}$ to $13 \pm 2.0 \mathrm{~mm} \mathrm{Hg}$ in all four patients.

\section{DISCUSSION}

Intra-abdominal pressure is an important physiological parameter that is still often neglected by the medical community [4]. It should be measured regularly in critically ill patients, 4 to 6-hourly, according to the guidelines [1]. According to the 2013 WSACS guidelines, IAH is defined

Table 3. Data from three consecutive days on IAH treatment

\begin{tabular}{|c|c|c|c|}
\hline & Day 1 & Day 2 & Day 3 \\
\hline Mean IAP (mm Hg) & $23(12-34)$ & $17.5(10-25)$ & $15(8-22)$ \\
\hline Mean APP $(\mathrm{mm} \mathrm{Hg})$ & 85.5 & 91.5 & 107 \\
\hline Mean SBP $(\mathrm{mm} \mathrm{Hg})$ & $108.5(83-134)$ & $109(90-128)$ & $122(101-143)$ \\
\hline Mean HR (beats $\min ^{-1}$ ) & $113(98-128)$ & $89.5(60-119)$ & $82(58-106)$ \\
\hline Mean Urinary Output (mL $\left.24 h^{-1}\right)$ & $1,500(400-2,600)$ & $1,105(310-1,105)$ & $1,200(0-2,400)$ \\
\hline Fluid Balance (last 24h) & $+2,160$ & $+1,730$ & $+2,931$ \\
\hline NGT tube need $(n)$ & 46 & 46 & 42 \\
\hline US gastric content observed ( $\mathrm{n})$ & 50 & 50 & 50 \\
\hline NGT observed on US (n) & 46 & 46 & 42 \\
\hline Positive bowel content (before enema) viewed on US ( $n$ ) & 50 & 50 & 50 \\
\hline Positive bowel content (after enema) viewed on US ( $n$ ) & 36 & 28 & 21 \\
\hline Bowel movements observed on US ( $n$ ) & 42 & 47 & 50 \\
\hline Number of patients with free abdominal fluid seen on US (n) & 27 & 24 & 23 \\
\hline Positive moderate to large amount of free abdominal fluid seen on US (n) & 6 & 6 & 4 \\
\hline US-guided paracentesis ( $\mathrm{n}$ ) & 2 & 0 & 0 \\
\hline
\end{tabular}


as a sustained increase in IAP equal to, or above $12 \mathrm{~mm}$ $\mathrm{Hg}$, that is frequently associated with abdominal (as well as extra-abdominal) pathology and complications [1, 5]. A missed IAH diagnosis can lead to longer ICU length of stay, prolonged ventilation, and higher incidence of ventilator-associated pneumonia, amongst other indirect consequences impairing patient recovery $[2,6]$. Therefore, it is paramount that ICU doctors and nurses are aware of the importance of IAH and ACS in both adults and children $[7,8]$. The presence of one or more risk factors for IAH should prompt appropriate IAP monitoring and help facilitate an early diagnosis. This monitoring should be included as a vital sign in the daily clinical evaluation of all critically ill patients.

The WSACS guidelines were updated in 2013, and included the Medical Management Algorithm as shown in Figure 1. These guidelines recommend either continuous or intermittent IAP monitoring. Medical management for $\mathrm{IAH}$ and ACS is divided into 5 categories:

1. Evacuation of intraluminal contents

2. Evacuation of intraluminal occupying lesions or extraluminal (intra-abdominal) contents

3. Improvement of abdominal wall compliance

4. Optimization of fluid administration

5. Optimization of systemic and regional perfusion Ultrasound is a useful adjunct in several of these medical management options.

Although POCUS has become an indispensable tool in the management of critically ill patients $[9,10]$, no research has been published on its use in IAH or ACS. There are currently few point-of-care bedside confirmation investigations that can confirm some of the clinical goals proposed by the WSACS, including nasogastric tube confirmation, assessment of colonic content, and OR evaluation of fluid removal from the abdomen. Based on this rationale, we have described the possibility of using POCUS in daily clinical practice, in the follow-up and treatment of critically ill patients with IAH/ACS diagnosis. We expanded the daily use of a portable Wi-Fi ultrasound device in patients with IAH/ACS in order to test the hypothesis that POCUS could be useful as an adjuvant treatment for IAH/ACS.

This study focused on the first two stages of the WSACS algorithm and the specific steps in each stage (escalating from 1 to 4). Our main objective was to use POCUS as an adjuvant tool for IAH management and thus focused our efforts on steps 1 to 3 of stage one ("evacuate intraluminal content"), and steps 1 and 2 of stage two ("evacuate intraluminal occupying lesions or extra-luminal content").

Most of the included patients were from the emergency department and were either taken to the operating room or intensive care unit. All admitted patients with risk factors for IAH had their IAP measured as a com- ponent of their vital signs every four to six hours. Diagnosis of IAH was made with three sustained IAP measurements over $12 \mathrm{~mm} \mathrm{Hg}$. All possible clinical steps were taken, according to current guidelines, to lower IAP once a measurement of IAP was found to be over $12 \mathrm{~mm} \mathrm{Hg}$. Eighty four percent of the enrolled patients were admitted on vasoactive drugs, with a mean systolic blood pressure of $108.5 \mathrm{~mm} \mathrm{Hg}$. This information was required to calculate the abdominal perfusion pressure [11]. As recommended by the World Society, abdominal perfusion pressure equals mean arterial pressure minus intra-abdominal pressure $(\mathrm{APP}=\mathrm{MAP}-\mathrm{IAP})$, and its measurement is mandatory for every IAP obtained. However, there is no available evidence investigating the utility of the above-mentioned formula in patients on high doses of vasoactive drugs. The use of vasoactive drugs and the effect on the systolic blood pressure may mask the significance of underlying intra-abdominal malperfusion, a consequence of vasoconstriction caused by the vasoactive agents. Therefore, the relatively normal APP may not accurately reflect intra-abdominal perfusion while the significance of these readings is not known. We suggest that the APP is not a reliable marker when measured in association with vasoactive drug usage [12]. Further research is necessary to investigate this hypothesis.

For patients with an IAP above grade I, decompression of intraluminal content is recommended. In this study the WSACS medical management algorithm (stage 1, step 1) was implemented accordingly with NGT insertion. The NGT was passed under direct US guidance with the probe on the epigastrium, allowing for direct visualization of the tip of the NGT as it was directed to its ideal position close to the pylorus (Fig. 2). A one-hundred-per cent accuracy was observed when using the US to determine NGT placement and positioning. US was also useful on the third ICU day when screening showed no gastric content and NGTs were removed in some patients. All included patients with gastric content viewed through the use of the US had prokinetics added to their prescription in accordance with $\mathrm{IAH}$ management.

POCUS was also used to evaluate bowel movements and colonic content. This helped daily assessment of post-operative patients and nutrition could be initiated earlier than usual in some cases due to detectable bowel movements. In these patients, although no bowel sounds were detected, bowel movement was detected with ultrasound. Likewise, POCUS was useful in detecting colonic material, thus guiding the physician on the need for further enemas to decompress the colon. These findings may also facilitate early recognition of bowel wall oedema, a consequence of extravasation from fluid resuscitation. In future, this may help early management of patient fluid balances [13]. 
Regarding the first 2 steps of the second stage of the WSACS medical management algorithm, ultrasound identified moderate to large amounts of free intra-abdominal fluid. These cases of cirrhotic patients with ascites required ultrasound-guided paracentesis. POCUS was also useful in patients with severe acute pancreatitis and IAH (Fig. 5A-C). Again, bedside ultrasound provided easy and prompt diagnosis and guided therapeutic management. All enrolled patients demonstrated reductions in IAP and subsequently better clinical performance during their first three days of admission. In Figure 6 we outline the role for the POCUS within the WSACS medical management algorithm.

Limitations of the study include a small sample size, different skill levels of ultrasound operators, and the observational design of the study. A randomized trial evaluating the clinical outcomes is required.

\section{CONCLUSION}

POCUS is a useful tool that should be used as an adjuvant in IAH management. It has the potential to be used in both diagnosis and treatment during the course of IAH, based on the Abdominal Compartment Society (WSACS) Guidelines.

\section{ACKNOWLEDGEMENTS}

1. This article is endorsed by the International Fluid Academy (IFA). The mission statement of the IFA is to foster education, promote research on fluid management and hemodynamic monitoring, and thereby improve the survival of the critically ill by bringing together physicians, nurses, and others from throughout the world and from a variety of clinical disciplines. The IFA is integrated within the not-for-profit charitable organization iMERiT, International Medical Education and Research Initiative, under Belgian law. The IFA website (http://www.fluidacademy.org ) is now an official SMACC affiliated site (Social Media and Critical Care) and its content is based on the philosophy of FOAM (Free Open Access Medical education — \#FOAMed). The site recently received the HONcode quality label for medical education (https://www.healthonnet.org/ HONcode/Conduct.html?HONConduct519739).

2. Conflicts of interest: Bruno Pereira is current President of WSACS (The Abdominal Compartment Society). Manu Malbrain is founding President and current Treasurer of WSACS, he is also member of the medical advisory Board of Pulsion Medical Systems (part of Maquet Getinge
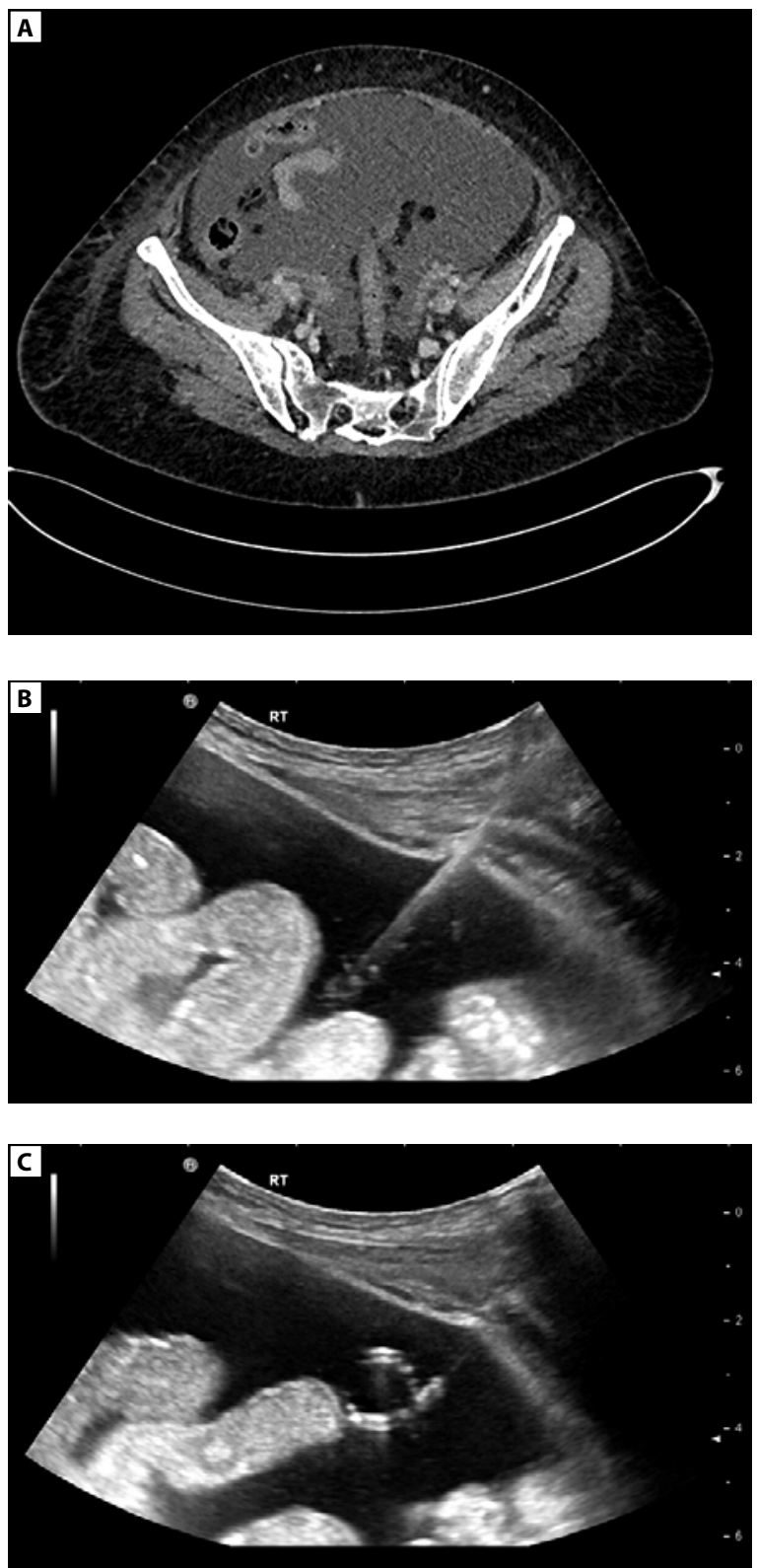

Figure 5. 75-year-old male with an IAP of $25 \mathrm{~mm} \mathrm{Hg}$ secondary to pancreatitis. A - axial contrast-enhanced abdominal CT demonstrates a large volume of ascites; $\mathbf{B}$ - US-guided abdominal drain insertion into a large fluid collection in the right flank; $\mathbf{C}-$ POCUS confirms the correct position of the pigtail catheter within the ascites fluid

group) and consults for ConvaTec, Acelity, Spiegelberg and Holtech Medical. Manu Malbrain is co-founder of the International Fluid Academy (IFA). The other authors have no possible conflicts of interest in relation to the content of this review article. 


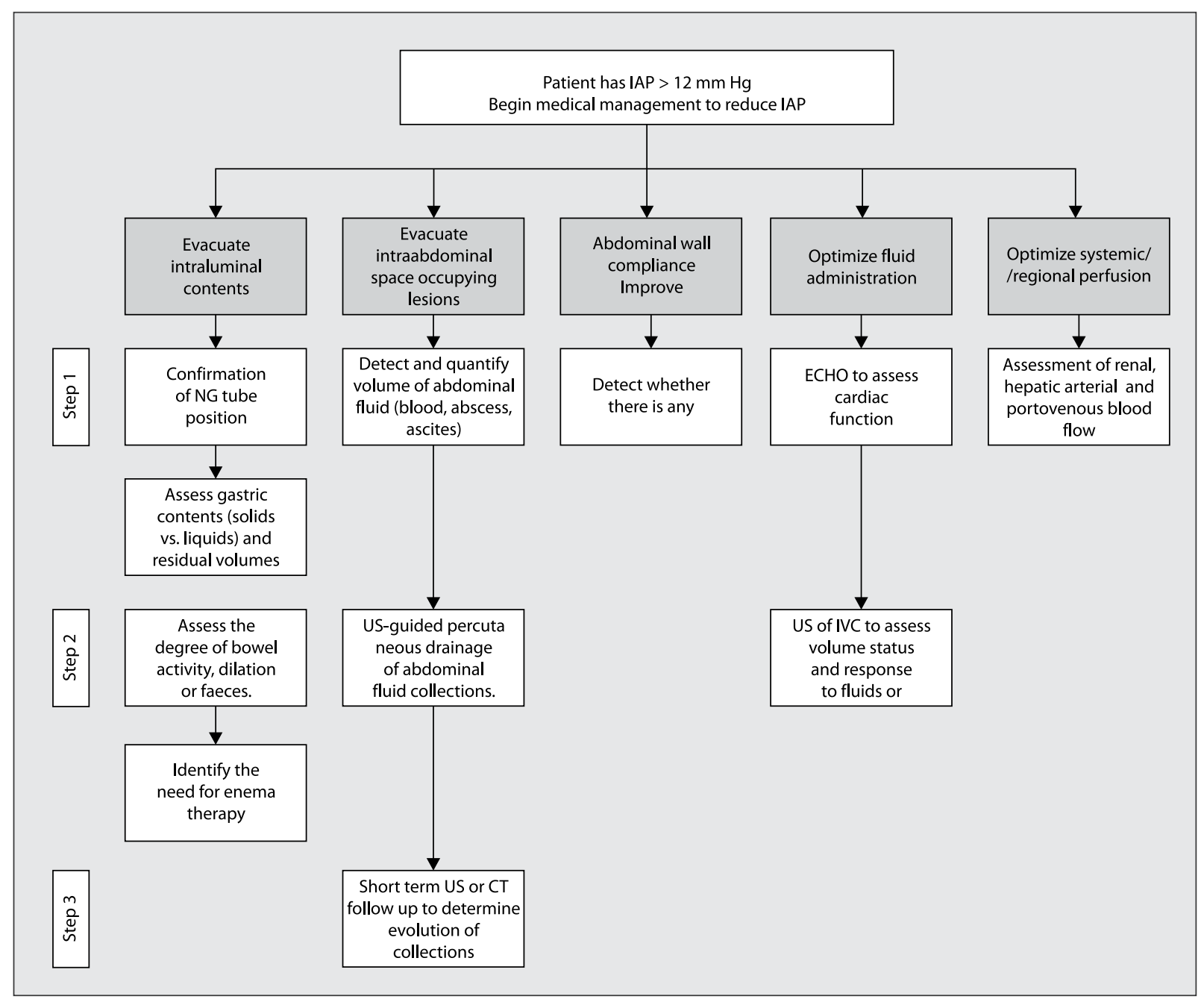

Figure 6. Role of POCUS within WSACS medical management algorithm

\section{References:}

1. Kirkpatrick AW, Roberts DJ, De Waele J, et al. Pediatric Guidelines Sub-Committee for the World Society of the Abdominal Compartment Syndrome. Intra-abdominal hypertension and the abdominal compartment syndrome: updated consensus definitions and clinical practice guidelines from the World Society of the Abdominal Compartment Syndrome. Intensive Care Med. 2013; 39(7): 1190-1206, doi: 10.1007/s00134-013-2906-z, indexed in Pubmed: 23673399.

2. Elbers $\mathrm{P}$, Rodrigus $\mathrm{T}$, Nossent $\mathrm{E}$, et al. Fluid therapy in critically ill patients: perspectives from the right heart. Anaesthesiol Intensive Ther. 2015; 47 Spec No: s38-s43, doi: 10.5603/AIT.a2015.0080, indexed in Pubmed: 26578396.

3. Lichtenstein D, van Hooland S, Elbers $P$, et al. Ten good reasons to practice ultrasound in critical care. Anaesthesiol Intensive Ther. 2014; 46(5): 323-335, doi: 10.5603/AIT.2014.0056, indexed in Pubmed: 25432552.

4. Wise R, Roberts DJ, Vandervelden S, et al. Awareness and knowledge of intra-abdominal hypertension and abdominal compartment syndrome: results of an international survey. Anaesthesiol Intensive Ther. 2015; 47(1): 14-29, doi: 10.5603/AIT.2014.0051, indexed in Pubmed: 25251947.

5. Malbrain ML, Chiumello D, Cesana BM, et al. WAKE-Up! Investigators. A Systematic review and individual patient data meta-analysis on intraabdominal hypertension in critically III patients: The Wake-Up Project World Initiative on Abdominal Hypertension Epidemiology, a Unifying Project (WAKE-Up!). Minerva Anestesiol. 2013 [Epub ahead of print]; 80(3)-306, indexed in Pubmed: 24336093.

6. Kirkpatrick AW, De Waele JJ, De Laet I, et al. WSACS - The Abdominal Compartment Society. A Society dedicated to the study of the physiology and pathophysiology of the abdominal compartment and its interactions with all organ systems. Anaesthesiol Intensive Ther. 2015; 47(3): 191-194, doi: 10.5603/AIT.a2015.0024, indexed in Pubmed: 25973657.

7. Divarci E, Karapinar B, Yalaz M, et al. Incidence and prognosis of intraabdominal hypertension and abdominal compartment syndrome in children. J Pediatr Surg. 2016; 51(3): 503-507, doi: 10.1016/j. jpedsurg.2014.03.014, indexed in Pubmed: 25783342.

8. Thabet FC, Bougmiza IM, Chehab MS, et al. Incidence, Risk Factors, and Prognosis of Intra-Abdominal Hypertension in Critically III Children: A Prospective Epidemiological Study. J Intensive Care Med. 2016; 31(6): 403-408, doi: 10.1177/0885066615583645, indexed in Pubmed: 25922384.

9. Ha YR, Toh HC. Clinically integrated multi-organ point-of-care ultrasound for undifferentiated respiratory difficulty, chest pain, or shock: a critical analytic review. J Intensive Care. 2016; 4: 54 doi: 10.1186/s40560-016-0172-1, indexed in Pubmed: 27529030.

10. Kameda T, Taniguchi N. Overview of point-of-care abdominal ultrasound in emergency and critical care. J Intensive Care. 2016; 4: 53, doi: 10.1186/s40560-016-0175-y, indexed in Pubmed: 27529029. 
11. Palakshappa JA, Meyer NJ. Which patients with ARDS benefit from lung biopsy? Chest. 2015; 148(4): 1073-1082, doi: 10.1378/ chest.15-0076, indexed in Pubmed: 25950989.

12. Cheatham $M$, White $M$, Sagraves $S$, et al. Abdominal perfusion pres sure: a superior parameter in the assessment of Intra-abdominal hypertension. The Journal of Trauma: Injury, Infection, and Critical Care. 2000; 49(4): 621-627, doi: 10.1097/00005373-200010000-00008.

13. Regli A, De Keulenaer B, De Laet I, et al. Fluid therapy and perfusional considerations during resuscitation in critically ill patients with intra-abdominal hypertension. Anaesthesiol Intensive Ther. 2015; 47(1): 45-53, doi: 10.5603/AIT.a2014.0067, indexed in Pubmed: 25421925.
Corresponding author:

Prof. Bruno M. Pereira

Av. Expedicionário Oswaldo

de Almeida Ramos, $n^{\circ} 280$

Centro Vassouras/RJ CEP: 27700-000, Brasil

e-mail:drbrunompereira@gmail.com

Received: 14.10 .2017

Accepted: 28.11.2017 